\title{
Venous thromboembolism in heart transplant recipients: incidence, recurrence and predisposing factors
}

\author{
Rolando J. Alvarez-Alvarez, MD, ${ }^{\mathrm{a}, \mathrm{b}}$ Eduardo Barge-Caballero, MD, PhD, ${ }^{\mathrm{a}, \mathrm{c}}$ Sergio A. \\ Chavez-Leal, MD, ${ }^{\mathrm{a}, \mathrm{b}}$ María J. Paniagua-Martin, MD, ${ }^{\mathrm{a}, \mathrm{c}}$ Raquel Marzoa-Rivas, MD, \\ $\mathrm{PhD},{ }^{\mathrm{a}, \mathrm{c}}$ Cayetana-Barbeito Caamaño, MD ${ }^{\mathrm{a}, \mathrm{c}}$ Ángela López-Sainz, MD, ${ }^{\mathrm{a}}$ Zulaika Grille- \\ Cancela, BS, ${ }^{a}$ Paula Blanco-Canosa, RN, ${ }^{a}$ José M. Herrera-Noreña, MD, ${ }^{\mathrm{c}, \mathrm{d}}$ José J. \\ Cuenca-Castillo, MD, ${ }^{\mathrm{c}, \mathrm{d}}$ Alfonso Castro-Beiras, MD, ${ }^{\mathrm{a}, \mathrm{c}}$ and María G. Crespo-Leiro, MD, \\ $\mathrm{PhD}^{\mathrm{a}, \mathrm{c}}$
}

From the ${ }^{a}$ Servicio de Cardiología. Complejo Hospitalario Universitario A Coruña, A Coruña, Spain; ${ }^{b}$ Fundación BBVA-Carolina, Madrid, Spain; ${ }^{c}$ Instituto de Investigación Biomédica de A Coruña (INIBIC), A Coruña, Spain; and the ${ }^{d}$ Servicio de Cirugía Cardiaca. Complejo Hospitalario Universitario A Coruña, A Coruña, Spain.

\begin{abstract}
Background. A high frequency of venous thromboembolism (VTE) has been observed after lung, kidney, and liver transplantation. However, data about the incidence of this complication among heart transplant (HT) recipients are lacking.

Methods. We analyzed the incidence, recurrence, and predisposing factors of VTE in a single-center cohort of 635 patients who underwent HT from April 1991 to April 2013. Deep venous thrombosis (DVT) and pulmonary embolism (PE) were considered as VTE episodes.

Results. During a median post-transplant follow-up of 8.4 years, 62 VTE episodes occurred in 54 patients $(8.5 \%)$. Incidence rates of VTE, DVT, and PE were, respectively, 12.7 (95\% confidence interval [CI], 9.7-16.3), 8.4 (95\% CI, 6.0-11.4), and 7.0 (95\% CI 4.8-9.7) episodes per 1,000 patient-years. Incidence rates of VTE during the first post-transplant year and beyond were, respectively, 45.1 (95\% CI, 28.9-67.1) and 8.7 (95\% CI 6.2-11.2) episodes per 1,000 patient-years. The incidence rate of VTE recurrence after a first VTE episode was 30.5 (95\% CI, 13.2-60.2) episodes per 1,000 patient-years. By means of multivariable Cox regression, chronic renal dysfunction, older age, obesity, and the use of mammalian target of rapamycin inhibitors were identified as independent risk factors for VTE among HT recipients.

Conclusions. VTE is a frequent complication after HT, mainly during the first post-operative year. In view of a high recurrence rate, long-term anti-coagulation should be considered in HT recipients who experience a first VTE episode.
\end{abstract}

Keywords:

Heart transplantation, Thromboembolism, Deep venous thrombosis, Pulmonary embolism 
Heart transplantation (HT) improves quality of life and survival in carefully selected patients with refractory heart failure. ${ }^{1}$ However, the life expectancy of HT recipients may be compromised by graftrelated complications, such as rejection and coronary allograft vasculopathy, as well as by comorbidities related to chronic immunosuppressive therapy, including infection, malignancy, diabetes mellitus, or renal dysfunction. ${ }^{2}$ Venous thromboembolism (VTE) is a less frequent but also relevant complication in some of these patients.

Solid-organ transplant recipients are exposed to an increased risk of VTE. ${ }^{3-14}$ In 2 large populationbased cohorts from United Kingdom ${ }^{15}$ and Canada, ${ }^{16}$ the estimated incidence rates of VTE among individuals from the general population were, respectively, 1.33 and 1.22 cases per 1,000 persons-years, decreasing to 1.07 cases per 1,000 persons-years when cancer-related VTE episodes were excluded. ${ }^{15}$ With variable follow-up duration among studies, the reported cumulative incidence of VTE varied between $4.5 \%$ and $9.1 \%$ in kidney transplant recipients, ${ }^{3-6}$ between $8.6 \%$ and $29 \%$ in lung transplant recipients, ${ }^{7-10}$ and between $0.4 \%$ and $4.6 \%$ in liver transplant recipients. ${ }^{11-13}$ Although comparing data from such heterogeneous studies is difficult, previous literature suggests that VTE is significantly more frequent in solid organ recipients than in individuals from the general population.

Major surgery, major trauma, prolonged bed rest, malignancy, obesity, renal dysfunction, thrombophilia, hormone replacement therapy, oral contraception, older age, and previous venous thromboembolism are consistent risk factors for VTE. ${ }^{17}$

To the best of our knowledge, only one published original paper ${ }^{14}$ has addressed the occurrence of VTE after HT. The authors of that $\operatorname{study}^{14}$ focused specifically on the role of sirolimus, a mammalian target of rapamycin (mTOR) inhibitor, as a risk factor for VTE. The purpose of our study was to describe the cumulative incidence and recurrence risk of VTE in HT recipients and to identify predisposing factors.

\section{Methods}

The Complejo Hospitalario Universitario A Coruña Institutional Review Board approved the study protocol.

\section{Setting and design of the study}

We conducted an observational study based on the historical cohort of patients aged $\geq 18$ years who underwent HT at the Complejo Hospitalario Universitario A Coruña (A Coruña, Spain) from April 1, 1991, to April 30, 2013. Our institution is a tertiary care university medical center with a reference population of $\sim 2.7$ million individuals and a routine activity of $\sim 25$ HT per year. Data for the study were extracted from a prospective database in which extensive clinical information has been recorded about all HT procedures performed at our institution since the beginning of our HT program in April 1, 1991.

\section{Follow-up protocol}

Patients were treated and monitored according to local protocols. During the early postoperative period, induction therapy with muromonab-CD3 or basiliximab was administered. The maintenance immunosuppressive regimen consisted of a combination of steroids, a calcineurin inhibitor (tacrolimus or cyclosporine A), and an anti-proliferative agent (azathioprine or mycophenolate mofetil). After the first post-transplant year, substitution of the anti-proliferative agent by an mTOR inhibitor (everolimus or sirolimus) was considered in selected patients with coronary allograft vasculopathy or recurrent cytomegalovirus infection. The introduction of an mTOR inhibitor was also considered in selected patients with malignancies or chronic renal dysfunction to minimize the exposure to calcineurin inhibitors. 
In our HT program, target serum levels of immunosuppressive drugs are 250 to $350 \mathrm{ng} / \mathrm{ml}$ ( $<6$ months after HT), 150 to $250 \mathrm{ng} / \mathrm{ml}(6-12$ months after HT), and 70 to $150 \mathrm{ng} / \mathrm{ml}$ (>1 year after HT) for cyclosporine A; 10 to $15 \mathrm{ng} / \mathrm{ml}(<1$ year after HT) and 5 to $10 \mathrm{ng} / \mathrm{ml}$ ( $>1$ year after HT) for tacrolimus; 3 to $8 \mathrm{ng} / \mathrm{ml}$ for everolimus and 6 to $15 \mathrm{ng} / \mathrm{ml}$ for sirolimus. Our institution first used mycophenolate mofetil in September 1997, sirolimus in February 1999, basiliximab in February 2001, and everolimus in March 2005.

Routine surveillance endomyocardial biopsies were performed periodically during the first 12 months after HT and afterwards only if rejection was clinically suspected. In stable patients, clinical visits, laboratory tests, 12-lead electrocardiogram, and transthoracic echocardiography were performed every 3 to 6 months after the first post-transplant year. Coronary angiographies were performed in case of clinical suspicion of coronary allograft vasculopathy, and, since 2003, also in all asymptomatic patients, barring contraindications, at 1 month, 1 year, and every 5 years after HT.

Thromboprophylaxis with sub-cutaneous low-molecular-weight heparins was recommended during hospital admissions in patients who required prolonged bed rest ( $>24$ hours), according to evolving practices guidelines. ${ }^{18}$ Specific regimens used in our institution included enoxaparin sodium (Clexane; Sanofi Aventis, Bridgewater, NJ), 2,000 to 4,000 IU daily; bemiparin sodium (Hibor; Laboratorios Farmacéuticos ROVI, S.A, Madrid, Spain) 2,500 to 3,500 IU daily, and nadroparin calcium (Fraxiparina; GlaxoSmithKline, Brentford, United Kingdom), 2,850 to 5,700 IU daily.

\section{Data collection and definition of variables}

The raw data extracted for the present analysis included variables regarding demographics (age, gender), pre-transplant clinical history (underlying disease leading to HT, cardiovascular risk factors, chronic renal dysfunction, supportive therapies), HT surgery (combined or redo transplantation, donor characteristics, surgical times, emergency transplantation), baseline immunosuppression, and posttransplant comorbidities (coronary allograft vasculopathy, malignancies). Chronic renal dysfunction was defined as the presence of a serum creatinine level $>2 \mathrm{mg} / \mathrm{dl}$ at least for 3 months before HT. The term "emergency HT" refers to the highest level of waiting list priority ("status 0"), according to the criteria of the organ donor allocation system in Spain, ${ }^{19}$ which requires the presence of refractory heart failure depending on mechanical circulatory support or intravenous inotropes, together with mechanical ventilation, or complicated with refractory recurrent ventricular arrhythmia.

Isolated deep venous thrombosis (DVT), isolated pulmonary embolism (PE), and simultaneous DVT plus PE were considered as VTE episodes. DVT was diagnosed by means of venous phlebography, venous echo Doppler, or computed tomography (CT) venography. PE was diagnosed by means of CT pulmonary angiogram, ventilation-perfusion nuclear scan, or autopsy. Complementary tests were driven by clinical findings and performed at the discretion of the attending physician, who was also responsible for determining the duration of oral anti-coagulation according to evolving practice guidelines. ${ }^{17}$ No specific diagnostic strategy was undertaken to identify silent VTE episodes in asymptomatic patients. Recurrence of VTE was defined as a new VTE episode occurring in a same patient at least 30 days after a previous one.

To investigate the occurrence of VTE episodes, study follow-up was extended from the date of transplantation until August 31, 2013, or until the date of death in the case of patients who died.

\section{Statistical analysis}

Categoric variables are presented as proportions, and continuous variables are presented as mean \pm standard deviation or median and interquartile range (IQR), depending on whether they were normally distributed. Clinical characteristics of patients who did and did not develop VTE after HT were compared by means of the chi-square test in the case of categoric variables and by the Student's $t$-student test in the case of continuous ones. 
Independent risk factors for VTE were assessed by means of backward stepwise Cox proportional hazards regression models. Because the incidence density rates of VTE during the first post-transplant year and after the first post-transplant year were markedly different, we designed specific multivariable models for each of these periods. Candidate variables that entered multivariable backward stepwise analyses were those that showed a univariable association with the study outcome with a $p$-value $<0.10$. All statistical comparisons were 2-tailed, and statistical significance was set as a $p$-value $<0.05$. Statistical analysis was performed with SPSS Statistics 20 software (IBM Corp., Armonk, NY).

\section{Results}

\section{Study population}

From April 1, 1991, to April 30, 2013, 635 patients, 107 (17\%) of whom were women, aged $\geq 18$ years underwent HT at our institution. Mean age was $54.6 \pm 12.4$ years. Transplantation was performed under a high-emergency status in 137 patients (22\%), $102(16 \%)$ of whom were on mechanical circulatory support and $81(13 \%)$ of whom were on mechanical ventilation.

Induction therapy was administered to 596 patients (94\%). Initial maintenance immunosuppression included steroids in 624 (98\%), tacrolimus in 98 (15\%), cyclosporine A in $489(77 \%)$, mycophenolate mofetil in $335(53 \%)$, azathioprine in $240(38 \%)$, sirolimus in $10(2 \%)$, and everolimus in 7 (1\%).

After HT, 122 patients (20\%) presented with malignancy, and $83(13 \%)$ had a diagnosis of coronary allograft vasculopathy. An mTOR inhibitor was initiated in 187 patients (29\%). Sirolimus was used in 59 (9\%), and everolimus in $128(20 \%)$.

\section{VTE episodes}

During a median post-transplant follow-up of 8.4 years (IQR, 3.3-13.2 years), 54 patients (8.5\%) experienced 62 VTE episodes. There were 28 isolated DVTs, 21 isolated PEs, and 13 instances of simultaneous DVT plus PE. Median time elapsed from HT to VTE was 2.6 years (IQR, 0.1-9 years).

The DVT diagnosis was established by means of venous phlebography in 22 cases, by means of echo Doppler in 15, and by CT venography in 4. Localizations of DVT were the lower limbs in 37, the inferior vena cava in 2 , the right upper limb in 1 , and the cavernous bodies in 1 . PE was diagnosed by means of CT pulmonary angiogram in 9 patients, by ventilation-perfusion nuclear scan in 20, and by autopsy in 5 .

Thirty VTE episodes (48\%) occurred within the first 30 days after a hospital admission. Reasons for hospitalization were HT in 11, endomyocardial biopsies in 4; 2 hospitalizations each for pancreatitis, respiratory infection, and heart failure; and 1 hospitalization each because of mediastinal infection, urinary tract infection, infectious colitis, carcinomatosis, acute coronary syndrome, coronary bypass grafting, inguinal hernia surgery, abdominal aneurysm surgery, and thoracic trauma.

Hospitalization involved prolonged ( $>24$ hours) bed rest in 25 patients; 18 of these patients $(72 \%)$ received evidence-based thromboprophylaxis. The remaining 32 VTE episodes (52\%) occurred in ambulatory patients with no previous hospitalization, and only $3(9 \%)$ were receiving evidence-based thromboprophylaxis at the time of VTE.

\section{Incidence of VTE}

The overall incidence rate of VTE in the study population was 12.7 (95\% confidence interval [CI], 9.7-16.3) episodes per 1,000 patient-years. Separated incidence rates of DVT and PE were, respectively, 8.4 (95\% CI 6.0-11.4) and 7.0 (95\% CI 4.8-9.7) episodes per 1,000 patient-years. 
During the first post-transplant year, 24 VTE episodes occurred in 22 patients (3.8\%), and after this, 38 VTE episodes were diagnosed in 35 patients (6.5\%). Incidence rates of VTE during the first posttransplant year and after the first post-transplant year were, respectively, 45.1 (95\% CI 28.9-67.1) and 8.7 (95\% CI 6.2-11.2) episodes per 1000 patient-years. Figure 1 shows the Kaplan-Meier estimates of the cumulative incidence of VTE in the first post-transplant year and during the entire follow-up period.
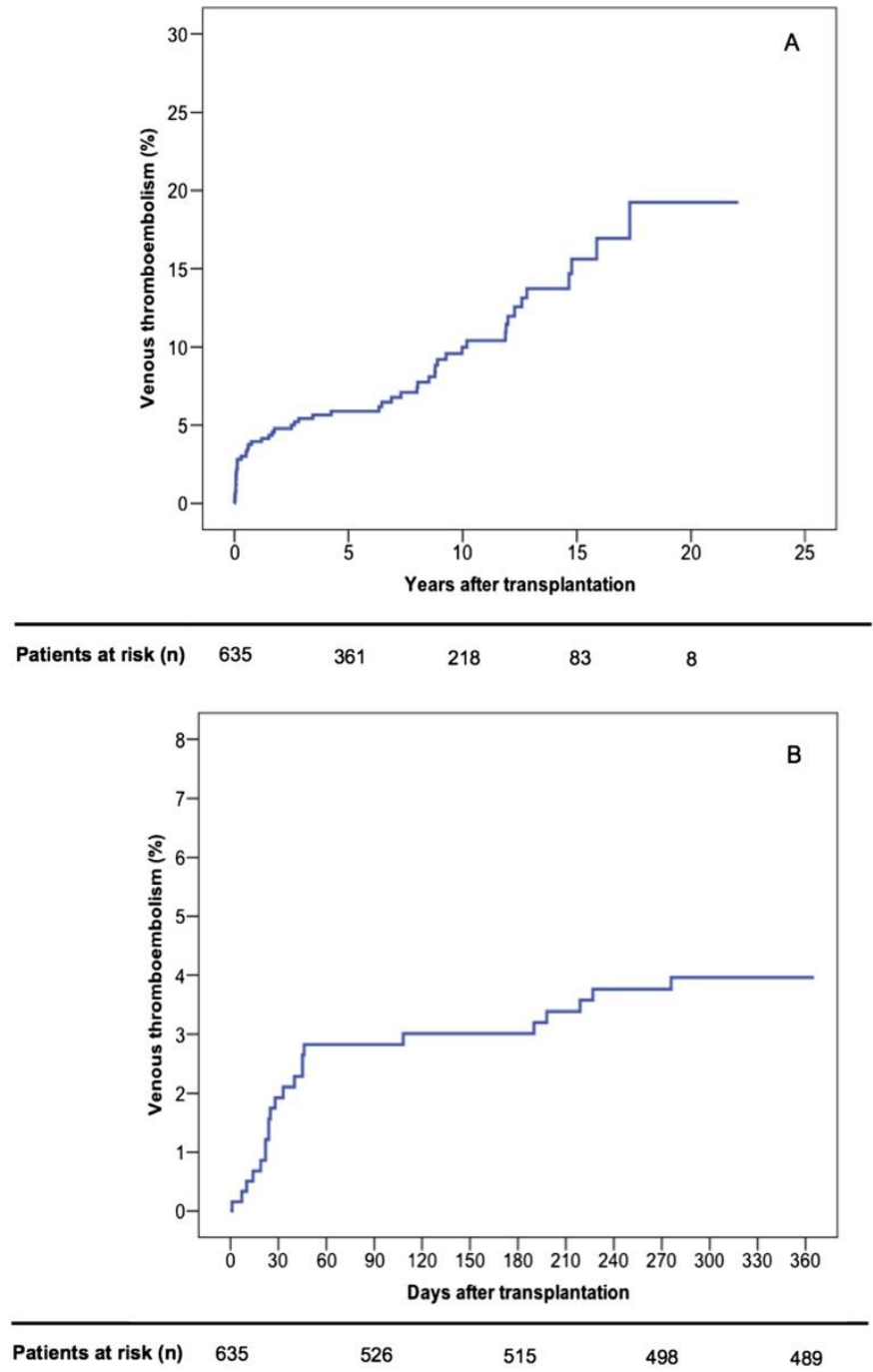

Figure 1. Kaplan-Meier estimates of the cumulative incidence of venous thromboembolism in the study population: (A) overall post-transplant followup and (B) 1-year post-transplant follow-up.

\section{Clinical characteristics of patients with or without VTE}

Table 1 reports the pre-transplant and post-transplant clinical cha racteristics of patients who did and did not present with a VTE episode. At the time of HT, VTE patients were older and had a higher prevalence of chronic renal dysfunction (serum creatinine $\geq 2 \mathrm{mg} / \mathrm{dl}$ ) and obesity (body mass index $\geq 30$ $\mathrm{kg} / \mathrm{m}^{2}$ ). After HT, malignancies and the use of mTOR inhibitors were more frequent among patients who developed VTE. 
Table 1. Clinical Characteristics of Patients With or Without Venous Thromboembolism

\begin{tabular}{|c|c|c|c|}
\hline \multirow{2}{*}{ Variables $^{\mathrm{a}}$} & \multirow{2}{*}{$\begin{array}{l}\text { VTE patients } \\
(n=54)\end{array}$} & \multirow{2}{*}{$\begin{array}{l}\text { Other patients } \\
(n=581)\end{array}$} & \multirow{2}{*}{$p$-value } \\
\hline & & & \\
\hline \multicolumn{4}{|l|}{ Pre-transplant clinical history } \\
\hline Age, years & $58.3 \pm 9$ & $54.3 \pm 12$ & 0.003 \\
\hline Women & $6(11)$ & $101(17)$ & 0.239 \\
\hline Reason for transplantation & & & 0.123 \\
\hline Ischemic heart disease & $29(54)$ & $230(40)$ & \\
\hline Dilated cardiomyopathy & $18(31)$ & $225(39)$ & \\
\hline Other & $7(15)$ & $134(21)$ & \\
\hline Diabetes & $7(13)$ & $87(15)$ & 0.691 \\
\hline Hypertension & $28(52)$ & $247(43)$ & 0.185 \\
\hline Hypercholesterolemia & $7(13)$ & $115(20)$ & 0.223 \\
\hline Obesity $^{\mathrm{b}}$ & $14(26)$ & $77(13)$ & 0.011 \\
\hline History of cigarette smoking & $28(52)$ & $257(51)$ & 0.918 \\
\hline Chronic renal dysfunction $^{c}$ & $9(17)$ & $49(8)$ & 0.045 \\
\hline Mechanical ventilation & $7(13)$ & $74(13)$ & 0.96 \\
\hline Mechanical circulatory support & $10(18)$ & $91(16)$ & 0.583 \\
\hline \multicolumn{4}{|l|}{ Heart transplant surgery } \\
\hline Second heart transplantation & 0 & $9(2)$ & 0.357 \\
\hline Combined transplantation & $1(2)$ & $23(4)$ & 0.44 \\
\hline Emergency transplantation & $11(20)$ & $126(22)$ & 0.82 \\
\hline Female donor & $6(11)$ & $101(17)$ & 0.239 \\
\hline Bypass time, min & $123 \pm 30$ & $126 \pm 48$ & 0.488 \\
\hline Cold ischemic time, min & $163 \pm 80$ & $190 \pm 77$ & 0.015 \\
\hline \multicolumn{4}{|l|}{ Initial immunosuppressive therapy } \\
\hline Induction therapy & $53(98)$ & $542(93)$ & 0.144 \\
\hline Steroids & $53(98)$ & $571(98)$ & 0.944 \\
\hline Cyclosporine & $45(83)$ & $434(75)$ & 0.159 \\
\hline Tacrolimus & $8(15)$ & $90(15)$ & 0.895 \\
\hline Mycophenolate mofetil & $21(39)$ & $314(54)$ & 0.033 \\
\hline Azathioprine & $31(57)$ & $209(36)$ & 0.002 \\
\hline Sirolimus & $2(4)$ & $8(1)$ & 0.189 \\
\hline Everolimus & 0 & $7(1)$ & 0.535 \\
\hline \multicolumn{4}{|l|}{ Post-transplant comorbidities } \\
\hline Coronary allograft vasculopathy & $13(19)$ & $73(13)$ & 0.214 \\
\hline Malignancy & $21(39)$ & $108(18)$ & $<0.001$ \\
\hline Switch to an mTOR inhibitor ${ }^{\mathrm{d}}$ & $30(56)$ & $157(27)$ & $<0.001$ \\
\hline
\end{tabular}

mTOR, mammalian target of rapamycin; VTE, venous thromboembolism.

${ }^{\text {a }}$ Continuous data are shown as the mean \pm standard deviation and categoric data as number $(\%)$.

${ }^{\mathrm{b}}$ Body mass index $\geq 30 \mathrm{~kg} / \mathrm{m}^{2}$.

${ }^{\mathrm{c}}$ Serum creatinine $\geq 2 \mathrm{mg} / \mathrm{dL}$.

${ }^{\mathrm{d}}$ Sirolimus or everolimus.

\section{Risk factors for VTE}

Multivariable Cox proportional hazards regression showed a statistically significant association between older age, chronic renal dysfunction, obesity, and the use of mTOR inhibitors with increased risk of VTE (Table 2). Post-transplant malignancy was associated with an increased risk of VTE in univariable analysis, but the statistical significance of this association was lost after multivariable adjustment. 
Table 2. Risk Factors for Venous Thromboembolism: Univariable And Multivariable Cox Proportional Hazards Regression Analysis $^{\mathrm{a}}$

\begin{tabular}{|c|c|c|c|c|}
\hline \multirow{2}{*}{ Variables } & \multicolumn{4}{|c|}{ Hazard ratio ( $95 \%$ confidence interval) } \\
\hline & Unadjusted & $p$-value & Adjusted & $p$-value \\
\hline \multicolumn{5}{|l|}{ Overall follow-up } \\
\hline Age, years & $1.04(1.01-1.07)$ & 0.012 & $1.04(1.01-1.08)$ & 0.017 \\
\hline Obesity $^{\mathrm{b}}$ & $2.30(1.25-4.24)$ & 0.007 & $1.88(1.001-3.52)$ & 0.050 \\
\hline Chronic renal dysfunction $^{c}$ & $2.20(1.07-4.49)$ & 0.031 & $2.06(1.01-4.23)$ & 0.048 \\
\hline Use of an mTOR inhibitor ${ }^{\mathrm{d}}$ & $2.08(1.21-3.59)$ & 0.008 & $1.87(1.07-3.27)$ & 0.029 \\
\hline Post-transplant malignancy & $1.81(1.04-3.52)$ & 0.035 & $1.49(0.84-2.61)$ & 0.179 \\
\hline \multicolumn{5}{|l|}{$<1$ year after transplantation } \\
\hline Age (years) & $1.11(1.03-1.18)$ & 0.003 & $1.12(1.05-1.20)$ & 0.001 \\
\hline Obesity $^{\mathrm{b}}$ & $2.81(1.15-6.90)$ & 0.024 & $2.71(1.09-6.73)$ & 0.032 \\
\hline Chronic renal dysfunction $^{c}$ & $3.12(1.15-8.45)$ & 0.025 & $2.83(1.03-7.77)$ & 0.043 \\
\hline Emergency transplantation & $3.52(1.52-8.13)$ & 0.003 & $5.49(2.35-12.83)$ & $<0.001$ \\
\hline \multicolumn{5}{|l|}{$>1$ year after transplantation } \\
\hline Obesity $^{\mathrm{b}}$ & $2.02(0.92-4.46)$ & 0.081 & $1.64(0.74-3.66)$ & 0.226 \\
\hline Use of an mTOR inhibitor ${ }^{\mathrm{d}}$ & $2.73(1.34-5.59)$ & 0.006 & $2.56(1.24-5.29)$ & 0.011 \\
\hline
\end{tabular}

mTOR, mammalian target of rapamycin.

${ }^{a}$ Variables shown in the Table are those that presented a univariable association with the study outcome with a $p$-value of $<0.10$.

${ }^{\mathrm{b}}$ Body mass index $\geq 30 \mathrm{~kg} / \mathrm{m}^{2}$.

${ }^{c}$ Serum creatinine $\geq 2 \mathrm{mg} / \mathrm{dl}$.

${ }^{\mathrm{d}}$ Sirolimus or everolimus.

Together with emergency HT, older age, chronic renal dysfunction, and obesity were identified as independent risk factors for VTE during the first post-transplant year, whereas the use of mTOR inhibitors was the unique variable that showed a statistically significant association with the occurrence of VTE after the first post-transplant year.

The incidence rate per 1,000 patient-years of VTE after the initiation of an mTOR inhibitor was 21.1 (95\% CI, 12.3-33.7) episodes, with a rate of 19.4 (95\% CI, 7.8-40.1) episodes in patients taking sirolimus, and 22.4 (95\% CI, 10.7-41.1) episodes in patients taking everolimus. The median time elapsed since the initiation of an mTOR inhibitor to the first VTE episode was 2.2 years (IQR, 0.3-3.1 years). At the time of VTE, median serum levels of mTOR inhibitors were $5.1 \mathrm{ng} / \mathrm{ml}$ (IQR, 3.7-6.1 ng/ml) in patients who were taking everolimus and $6.3 \mathrm{ng} / \mathrm{ml}(\mathrm{IQR}, 4.2-9.1 \mathrm{ng} / \mathrm{ml})$ in patients who were taking sirolimus.

\section{Treatment}

Among 54 patients with a first VTE episode, 4 (7.4\%) died within the first 24 hours after clinical presentation. Autopsy confirmed PE as a major contributor cause of death in all of these patients. The remaining 50 patients were treated with different anti-coagulant strategies. Upon diagnosis, lowmolecular weight heparin was initiated in 26 patients (52\%) and intravenous heparin in 24 (48\%). In addition, 1 patient received intravenous thrombolysis, 1 patient underwent inferior vena cava filter implantation, and 1 patient underwent venous embolectomy.

After the early post-VTE phase, 47 patients (94\%) received oral acenocoumarol (Sintrom, Novartis Pharma, Basel, Switzerland), with an international normalized ratio (INR) therapeutic target of 2 to 3 . No patient was treated with rivaroxaban or apixaban. Oral anti-coagulation was discontinued in 29 patients (58\%), 3 of them due to major bleeding, and was maintained permanently until the end of follow-up in 21 patients $(42 \%)$. The median duration of anti-coagulant therapy in the entire population was 515 days 
(IQR, 192-1,448 days). The median duration of anti-coagulation in patients who discontinued this treatment was 252 days (IQR, 101-510 days).

\section{Recurrence}

Recurrence of VTE was observed in 8 patients (16.3\%), 1 of whom died as a consequence of the relapsing episode (PE). Median time elapsed from the first VTE episode to VTE recurrence was 1.7 years (IQR, 0.6-8 years). The overall incidence rate of VTE recurrence after a first VTE episode was 30.5 (95\% CI, 13.2-60.2) episodes per 1,000 patient-years.

Only 2 patients (4\%) experienced a VTE recurrence while on anti-coagulant therapy. The VTE recurrence in 1 patient appeared during a transient discontinuation of the treatment due to a planned invasive procedure. Incidence rate of VTE recurrence per 1,000 patient-years was 19.2 (95\% CI, 2.3$69.5)$ episodes in patients receiving anti-coagulation and 50.8 (95\% CI, 18.7-110.7) episodes after the permanent discontinuation of this therapy.

\section{Discussion}

This study systematically assessed the incidence, recurrence, and predisposing factors of VTE after HT. In our single-center cohort of 635 consecutive HT recipients with a median follow-up of more than 8 years, we observed a VTE cumulative incidence of $8.5 \%$ and a VTE incidence rate of 12.7 episodes per 1,000 patient-years. The risk of VTE was higher during the first 12 months after HT (45.1 episodes per 1,000 patient-years) and decreased significantly beyond this time (8.7 episodes per 1,000 patient-years). Even if VTE episodes that occurred within the first post-transplant year are not taken into account, these data reflect more than a 6-fold increase of VTE incidence compared with that reported for individuals from the general population. ${ }^{15,16}$ After a first VTE episode, the risk of recurrence was high (30.5 episodes per 1,000 patient-years), especially after the discontinuation of anti-coagulant therapy (50.8 episodes per 1,000 patient-years). By means of multivariable models, chronic renal dysfunction, older age, obesity, and an emergency transplantation were identified as independent risk factors for early $(<1$ year) posttransplant VTE, whereas the use of mTOR inhibitors was identified as an independent risk factor for the occurrence of late (>1 year) post-transplant VTE.

Solid-organ transplant recipients are exposed to a higher risk of VTE than patients from the general population. With variable follow-up duration among studies, the reported cumulative incidence of VTE varied between $4.5 \%$ and $9.1 \%$ in kidney transplant recipients, ${ }^{3-6}$ between $8.6 \%$ and $29 \%$ in lung transplant recipients, ${ }^{7-10}$ and between $0.4 \%$ and $4.6 \%$ in liver transplant recipients. ${ }^{11-13}$ In a single-center observational study ${ }^{14}$ including 67 HT recipients treated with a sirolimus-based immunosuppressive regimen and 134 HT matched controls, the cumulative incidence of VTE during a mean follow-up of more than 3 years was $8.5 \%$. Hypercoagulability secondary to chronic immunosuppressive therapy is thought to play an important role in the increased thromboembolic risk of solid-organ transplant recipients. ${ }^{7,20-21}$

Almost $40 \%$ of all VTE episodes in our series occurred within the first 12 months after HT, most of them during the early post-operative period. Previous studies ${ }^{3,8}$ have already shown that the risk of VTE is high at early stages after solid-organ transplantation, favored by surgical injury and immobilization. In our study, obesity, older age, and chronic renal dysfunction were associated with an increased incidence of early post-transplant VTE. These are well-characterized pre-disposing factors for VTE in other solidorgan recipients ${ }^{3,9,13}$ and also in the general population. ${ }^{22-24}$ Emergency transplantation was also identified as a strong risk factor for early post-transplant VTE. The pre-transplant clinical condition in patients undergoing this type of procedure is usually severely impaired and requires invasive therapies, such as mechanical circulatory support or mechanical ventilation, and they are exposed to an increased risk of post-transplant complications such as primary graft failure, nosocomial infection, and critical illness polyneuropathy. This clinical picture frequently results in prolonged stays in the intensive care unit and in late postoperative mobilization, which increase the risk of VTE. 
The use of mTOR inhibitors (sirolimus and everolimus) was the only variable that showed a statistically significant association with the occurrence of VTE after the first post-transplant year in our cohort. A few recent studies have suggested that solid organ recipients receiving mTOR inhibitors might be exposed to an increased risk of thromboembolic events, raising a significant concern about the safety of this group of drugs. A sub-analysis of a randomized clinical trial ${ }^{10}$ found that lung transplant recipients treated with a sirolimus-based regimen presented a statistically significant higher cumulative incidence of VTE during a 3-year follow-up period of $17.2 \%$ vs $3.2 \%$ in others treated with an azathioprine-based regimen. In an observational study, ${ }^{14}$ HT recipients on a sirolimus-based regimen presented a higher cumulative incidence of VTE than matched controls not receiving mTOR inhibitors (12\% vs 7\%), but this difference was no longer statistically significant after multivariable adjustment. A study in renal transplant recipients ${ }^{25}$ revealed that patients treated with everolimus had higher serum levels of several pro-coagulant factors compared with patients treated with other immunosuppressive regimens, resulting in increased endothelial activation, enhanced thrombin formation, and impaired fibrinolysis. However, the evidence that supports a potential association between mTOR inhibitors and an increased risk of VTE events is still weak and might be confounded by a high prevalence of comorbid conditions such as chronic renal failure, dyslipidemia, or malignancy in patients taking these kinds of drugs. Larger prospective multicenter studies are therefore warranted to clarify this question.

Another key point of our investigation is the significant risk of VTE recurrence observed in HT recipients who experienced a first VTE episode, consistently with previously described in renal transplant recipients. ${ }^{4-5}$ Of note, most of the recurrent VTE episodes occurred in patients in whom oral anticoagulation had already been stopped. In view of these findings, it might be reasonable to maintain longterm oral anti-coagulation in HT recipients who experience a first VTE episode, especially when any other permanent risk factor is present, such as obesity, malignancy, renal dysfunction, mTOR inhibitor therapy, and provided that the estimated bleeding risk is not excessively high.

Our study has a few limitations. First, we conducted a retrospective analysis, so results may be conditioned by selection, information, and confusion biases inherent to this type of investigation. The relatively low number of VTE episodes after the first post-transplant year may have prevented us from detecting a statistically significant association between certain variables and the study outcome, as might be the case with post-transplant malignancy. Also, the association between the use of mTOR inhibitors and VTE must be interpreted with caution, given that the study design did not allow us to establish a conclusive cause-and-effect correlation between both variables. Data on international normalized ratios were not collected, so we are not able to extract reliable conclusions about the efficacy of anti-coagulant therapy in the HT population. Finally, the limited setting of the investigation, which was conducted in a single institution, implies that its external validity is not warranted.

In conclusion, VTE is a relatively frequent complication in HT recipients, especially during the first post-transplant year. In our study, classic thromboembolic risk factors, such as older age, obesity, and renal dysfunction, were associated with increased risk of early $(<1$ year) post-transplant VTE, whereas the use of mTOR inhibitors was a major predisposing factor for late ( $>1$ year) post-transplant VTE. Interestingly, the risk of VTE recurrence after a first VTE episode was high, so our opinion is that longterm oral anti-coagulation should be maintained in these patients, especially if other risk factors are present and provided that the bleeding risk is not excessive. Further investigation is required to clarify the underlying mechanisms and the optimal clinical management of venous thromboembolic complications in this specific population.

\section{Disclosure statement}

Financial support for this investigation was received from the Fundación BBVA-Carolina through a research grant awarded R.J.A.-A.

E.B.-C., R.M.-R., M.J.P.-M., and M.C.-L. have received travel grants from Novartis Pharma and Astellas Pharma. None of the other authors has a financial relationship with a commercial entity that has an interest in the subject of the presented manuscript or other conflicts of interest to disclose. 


\section{References}

1. Costanzo, M.R., Dipchand, A., Starling, R. et al. The International Society of Heart and Lung Transplantation Guidelines for the care of heart transplant recipients. J Heart Lung Transplant. 2010; 29: 914-956

2. Lund, L.H., Edwards, L.B., Kucheryavaya, A.Y. et al. The Registry of the International Society for Heart and Lung Transplantation: thirtieth official adult heart transplant report-2013; focus theme: age. J Heart Lung Transplant. 2013; 32: 951-964

3. Humar, A., Johnson, E.M., Gillingham, K.J. et al. Venous thromboembolic complications after kidney and kidney-pancreas transplantation: a multivariate analysis. Transplantation. 1998; 65: 229-234

4. Poli, D., Zanazzi, M., Antonucci, E. et al. High rate of recurrence in renal transplant recipients after a first episode of venous thromboembolism. Transplantation. 2005; 80: 789-793

5. Zanazzi, M., Poli, D., Antonucci, E. et al. Venous thromboembolism in renal transplant recipients: high rate of recurrence. Transplant Proc. 2005; 37: 2493-2494

6. Poli, D., Zanazzi, M., Antonucci, E. et al. Renal transplant recipients are at high risk for both symptomatic and asymptomatic deep vein thrombosis. J Thromb Haemost. 2006; 4: 988-992

7. Izbicki, G., Bairey, O., Shitrit, D., Lahav, J., and Kramer, M.R. Increased thromboembolic events after lung transplantation. Chest. 2006; 129: 412-416

8. Kahan, E.S., Petersen, G., Gaughan, J.P., and Criner, G.J. High incidence of venous thromboembolic events in lung transplant recipients. J Heart Lung Transplant. 2007; 26: 339-344

9. Yegen, H.A., Lederer, D.J., Barr, R.G. et al. Risk factors for venous thromboembolism after lung transplantation. Chest. 2007; 132: 547-553

10. Ahya, V.N., McShane, P.J., Baz, M.A. et al. Increased risk of venous thromboembolism with a sirolimus-based immunosuppression regimen in lung transplantation. J Heart Lung Transplant. 2011; 30: 175-181

11. Cherian, T.P., Chiu, K., Gunson, B. et al. Pulmonary thromboembolism in liver transplantation: a retrospective review of the first 25 years. Transpl Int. 2010; 23: 1113-1119

12. Sakai, T., Matsusaki, T., Dai, F. et al. Pulmonary thromboembolism during adult liver transplantation: incidence, clinical presentation, outcome, risk factors, and diagnostic predictors. Br J Anaesth. 2012; 108: 469-477

13. Salami, A., Qureshi, W., Kuriakose, P., Moonka, D., Yoshida, A., and Abouljoud, M. Frequency and predictors of venous thromboembolism in orthotopic liver transplant recipients: a single-center retrospective review. Transplant Proc. 2013; 45: 315-319

14. Thibodeau, J.T., Mishkin, J.D., Patel, P.C. et al. Sirolimus use and incidence of venous thromboembolism in cardiac transplant recipients. Clin Transplant. 2012; 26: 953-959

15. Martinez, C., Cohen, A.T., Bamber, L., and Rietbrock, S. Epidemiology of first and recurrent venous thromboembolism: a population-based cohort study in patients without active cancer. Thromb Haemost. 2014; 112: $255-263$

16. Tagalakis, V., Patenaude, V., Kahn, S.R., and Suissa, S. Incidence of and mortality from venous thromboembolism in a real-world population: the Q-VTE Study Cohort. Am J Med. 2013; 126: e13-e21

17. Blann, A.D. and Lip, G.Y. Venous thromboembolism. BMJ. 2006; 332: 215

18. Gould, M.K., Garcia, D.A., Wren, S.M. et al. American College of Chest Physicians. Prevention of VTE in nonorthopedic surgical patients: Antithrombotic Therapy and Prevention of Thrombosis, 9th ed: American College of Chest Physicians Evidence-Based Clinical Practice Guidelines. (e227-77S)Chest. 2012; 141

19. Barge-Caballero, E., Segovia-Cubero, J., Almenar-Bonet, L. et al. Preoperative INTERMACS profiles determine postoperative outcomes in critically ill patients undergoing emergency heart transplantation: analysis of the Spanish National Heart Transplant Registry. Circ Heart Fail. 2013; 6: 763-772

20. Stahl, R.L., Duncan, A., Hooks, M.A., Henderson, J.M., Millikan, W.J., and Warren, W.D. A hypercoagulable state follows orthotopic liver transplantation. Hepatology. 1990; 12: 553-558

21. Velasco, F., Villalba, R., Fernandez, M. et al. Diminished anticoagulant and fibrinolytic activity following liver transplantation. Transplantation. 1992; 53: 1256-1261

22. Stein, P.D., Beemath, A., and Olson, R.E. Obesity as a risk factor in venous thromboembolism. Am J Med. 2005; 118: 978-980

23. Cosmi, B., Legnani, C., Tosetto, A. et al. Sex, age and normal post-anticoagulation D-dimer as risk factors for recurrence after idiopathic venous thromboembolism in the Prolong study extension. J Thromb Haemost. 2010; 8: $1933-1942$

24. Wattanakit, K., Cushman, M., Stehman-Breen, C., and Heckbert, S.R. Folsom AR. Chronic kidney disease increases risk for venous thromboembolism. J Am Soc Nephrol. 2008; 19: 135-140

25. Baas, M.C., Gerdes, V.E., Ten Berge, I.J. et al. Treatment with everolimus is associated with a procoagulant state. Thromb Res. 2013; 132: 307-311 